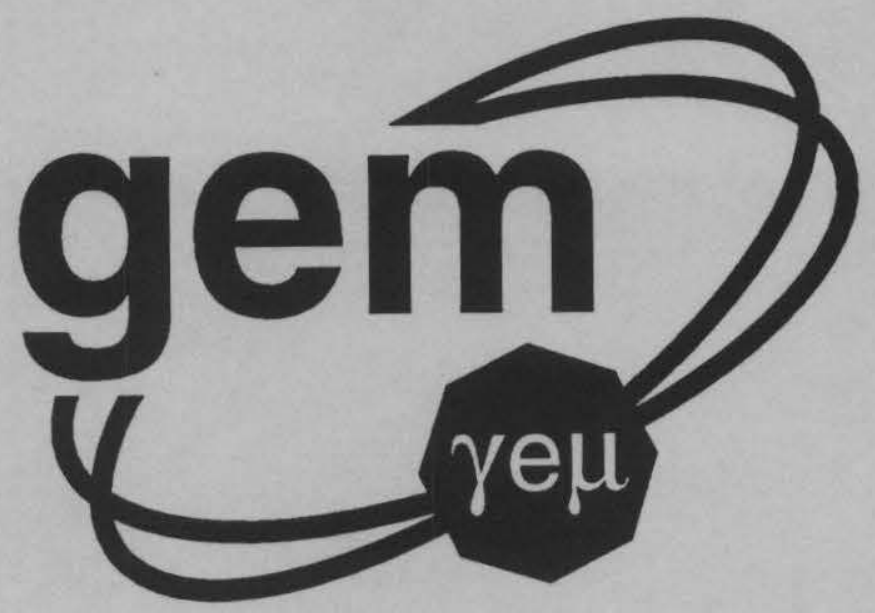

\title{
High Precision Straw Tube Chamber With Cathode Readout
}

V. N. Bychkov, I. A. Golutvin, Yu. V. Ershov, E. V. Zubarev, A. B. Ivanov, V. N. Lysiakov, A. V. Makhankov, S. A. Movchan, V. D. Peshekhonov, T. Preda Joint Institute for Nuclear Research

\section{Abstract:}

The high precision straw chamber with cathode readout was constructed and investigated. The $10 \mathrm{~mm}$ diameter straws were made of aluminized mylar with transparent longitudinal window. The $\mathrm{X}$ coordinate information has been taken from the cathode strips as induced charges and investigated by centroid method. The spatial resolution $\sigma_{\mathrm{x}}=120 \mu \mathrm{m}$ was obtained at the signal/noise ratio about 60 . The possible ways to improve the signal/noise ratio is discussed. 


\section{JOINT INSTITUTE FOR NUCLEAR RESEARCH \\ PARTICLE PHYSICS LABORATORY}

\section{HIGH PRECISION STRAW TUBE CHAMBER WITH CATHODE READOUT}

V.N.Bychkov, I.A.Golutvin, Yu.V.Ershov, E.V.Zubarev, A.B.Ivanov, V.N.Lysiakov, A.V.Makhankov, S.A.Movehan, V.D.Peshekinonov, T.Preda

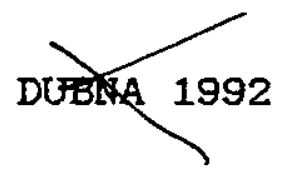




\begin{abstract}
The high precision straw chamber with cathode readout was constructed and invezigated. The $10 \mathrm{~mm}$ diameter straws were made of aluminized mylar with transparent iongitudinal window. The $X$ coorinate intomation has been taken from the cathode strips as induced charges and investigated by centroid method. The spatial resoiution $\sigma_{x}=120 \mathrm{\mu m}$ was obtained at the signal/noise ratio about 60 . The possible ways to improve the signal,noise ratio, is discussed.
\end{abstract}




\section{Introduction}

Iuring the iszt yejrs nex netizications of gas

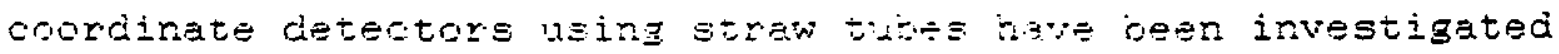
and Froposed as innor trocking oystems for LHC and SSC experimente [1]. The stron charhers rave high frecision ( 50 (im), high rate capabilitiss, short memory time and can work with high pressure up to 4 atm. They can be industrially producted and used also as a part of large-somle muon detecting systems.

In this paper we present characteristics of the straw chamber with myiar straws and cathode readout which combines to some extend propertils of the drift chambers [2]. drift metal tubes [3]. noneyoomb chambers with cathode readout $[4]$.

\section{The chamber construction}

The scheme of the chamber is shown in fig.1. It consists of a set of mylen straws fixed to the fiberglass auport. Un the upper surface of the support there are copper strips, orthosonjl to straws. Thidiness of fiberslass plane and copoer stripe is $2.5 \mathrm{~mm}$ and $50 \mathrm{\mu m}$ respetively. Strip pitch $(a+b)$ is 3,4 or $5 \mathrm{~mm}$ and interval value b is $0.5,0.5$ or $1 \mathrm{~mm}$, respectively. The length of the strigs was $1 \mathrm{~m}$.

The straws, $10 \mathrm{~mm}$ in diameter with longitudinal sear., were made of the aluminized mylar 25 lm thick processed by ulirasonic weiding. In our construction the straws with aluminiunless longitudinal windowe facing toward the strips have been used. The gold flated tungsten wires are kept with 200 grams tension in the center of the etraw. The wire diameters are 50 Hm. The tube body tension is 100 grams.

With this chamber one can determine Y-coordinates of the charged particle tracks by drift time measurement, and $X$-coordinates by processing the charges which are induced by avalanches on the strips through the transparent window. 


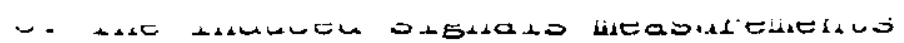

The maximum velue of tro wandow angle, gxp, is limited by the value of the accumizati electrical charge. Eig. 2 gives the dependence of the reletive anode signal on the window size. The measurements were done with 8 keV X-ray beam of $i \mathrm{~mm}$ in diameten. The beam intensity was $2 \times 10^{4}$ $\mathrm{sm}^{-2} \mathrm{~s}^{-1}$ and the $g \equiv \mathrm{g}$ gin was about $2 \times 1 \mathrm{~g}^{5}$. For window angles more than $36^{\circ}$ the anode eignal drops very fest. This size was chosen for further measurements.

The distribution of the induced charge in $\mathrm{X}$-direction $i=$ given in fig. 3 for $13 \mathrm{k} \Omega$ and $290 \mathrm{k} \Omega$ input resistance of the cherge amplifiers. The total width of the distribution (FWTM) is almost independent of the input resistance and is about $3 \mathrm{R}$, where $R$ is the straw radius. Together with [6], we conclude that the optimum strip pitch should be equal to $R$ for the best spetial resolution.

Figure 4 shows the distribution of the indueed signals on a 200 fun diameter wire moving parallel to the enode wire in Eront of the transparent, window. The angle $\vartheta$ has vertex on the anode wire and $\theta=0$ correspords to the middile of the window. The widih of the distribution (EWTM) correspondes to the window size.

\section{The spatial resolution}

The spatial resolution of the chamber has been investigated using the X-rays collimated beam moving with good accuracy in $X$ and $Y$ directions. The beam diameter was less than $105 \mathrm{~km}$ (EWHM). The signals from the strips through the charge-sensitive amplifier wers transported to the TuC and then to FC computer. The anode signais trigger the system. The amplicier parameters are:

- input resistance is $1.3 \mathrm{k} \Omega$;

- voltage-charge conversion coefficient is $6.7 \mathrm{~V} / \mathrm{pC}$;

- noise is $3 * 19^{-3}$ po (60);

- integrating time is $60 \mathrm{~ns}$.

The gas mixture was Ar/CH4(59,50).

The spatial resolution is determined by centroid $[6,7]$ and charge-ratio methods [4] using charge read out from 


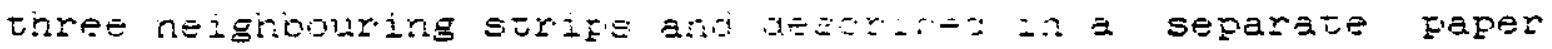

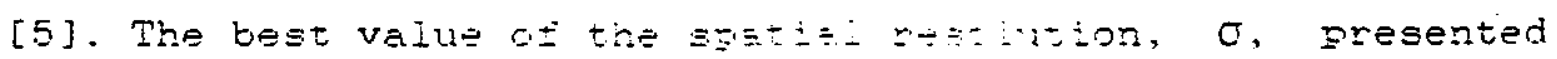

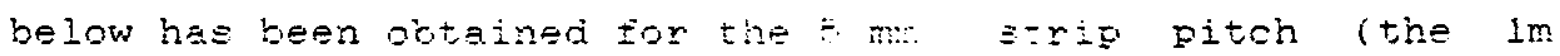
length strif had eapacity about 3 do fi.

we have obtained the spatial resolution, 0 , fitting the event distributions measursi with the different signai,noise ratios. An exampit of these distributions is shown in tig. 5 .

Figure 6 presents the dependence of the spatial resolution on the siznal,roise ratio for the avalanche position near the center of the strip. We have cheched that it is ratriar independent on the avalanche position across the strifs. One can see irom fig. 6 that the spatial resolution almost lineariy defends on the signal/noise ratio. The best resolution of (193 $194 \mathrm{Hm}$ is obtained at the signal, noise ratio of about 70 . This can be further reduced using higher signalinoise ratios. There are severel ways to improve this ratio probably by factor two [B]:

- using low-noise electronise;

- working with higher gas gain (in the saturated proportional mode and limited streamer mode);

- using the myler straws with carbon covering the window.

\section{Conclusion}

In conolusion we have constructed and investigated charateristics of the straw chamber with catiode strip $r=a t o u s$.

The spatial resolution of (10.3 4) pim was obtained with the signel/noise ratio at about 70 . This value is good enough for a single layer of the Lido soc muon detector.

The authors express their gratitude to V.s. Khabarov for his heip during the work and Z.I. Sminnova for assembling quality. 


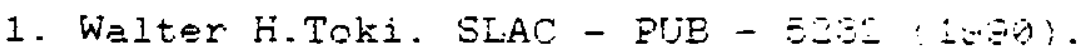

2. B.Adeva, M.Aguilar-Benteez, H.Akberi et ai. Nuel. Instr.and Meth. in Physios Research A289 (1990) 35.

3. GEM. Letters of Intent.

4. H.van der Grazf, J.Bushens, G.Fabez et al. Nucl. Instr.and Meth. in Physics Research A:387 (1991) 220.

5. I.A. Golutvin, S.A. Movchan, V.D. Peshechonov, T. Preda, to be published in Nucl. Instr. and Meth.

6. I.Endo, T.Kawamoto, Y.Mizuno et al. Nucl. Instr.and Meth. 188 (1981) 51 .

7. J.Chiba, H.Iwasaki, T.Kageyama et al. Nucl. Instr.and Meth. 206 (1983) 451.

8. V.D.Pestekhonov. Wire Chamber Conference 1992, Viena, Austria (February, 1992) (to be published). 


\section{FIGURE CAPTIONS}

Fig.1. The soheme of the chamber (at right) and the straw (at left).

Fig.2. The degendence of the relative anode signal on window angle.

Fig.3. The spatial distribution of induced charge value on Xcoordinate.

Fig.4. The indueed charge distribution across the straw window.

FiE.5. The event distribution as a function of the avaianche Fosition messured with signal/moise ratio of about 60 . The soilde line is gaussian fit.

Fis.6. The detector spatial resolution dependence on signal, noise ratio. 


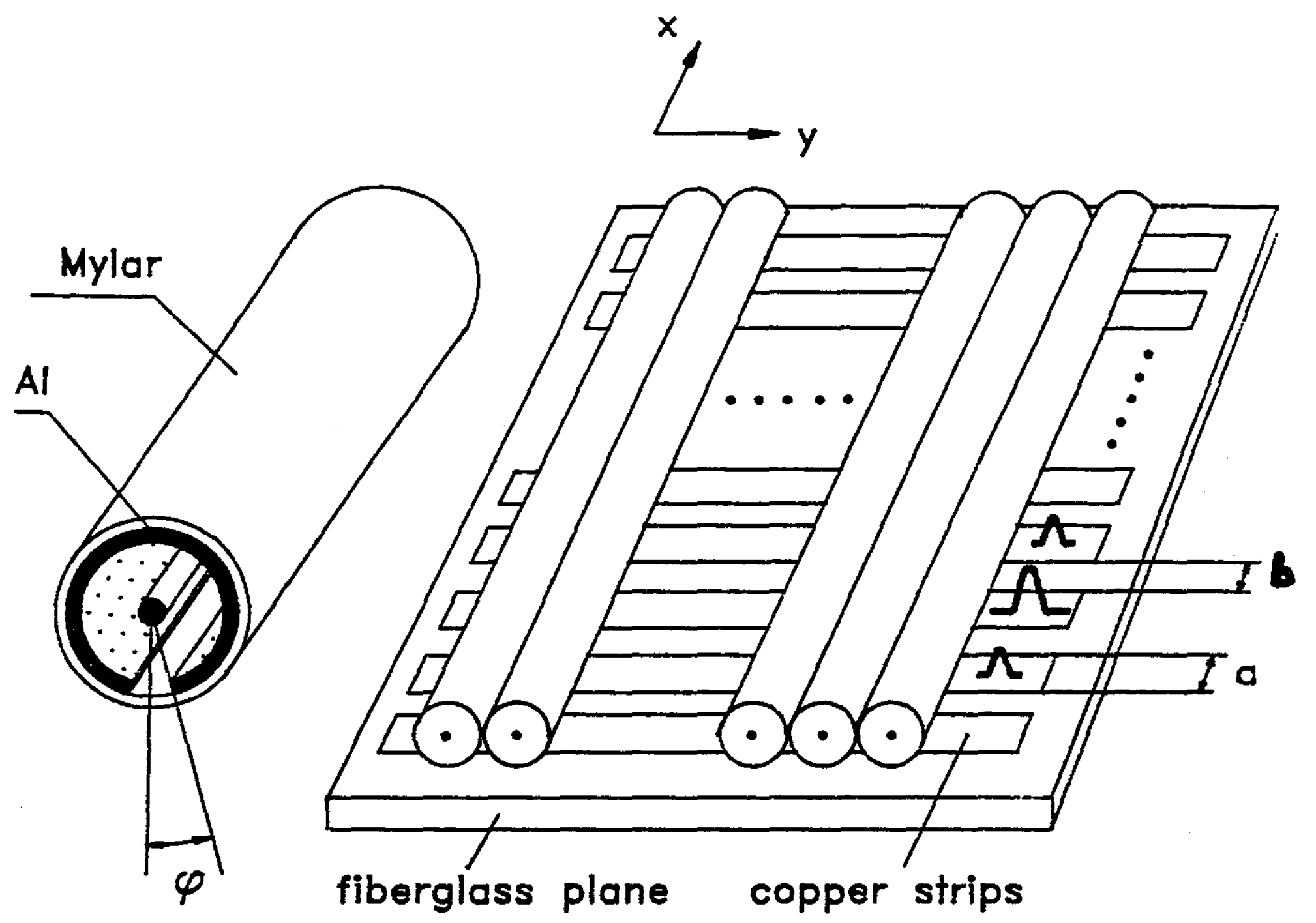

Fig. 1 


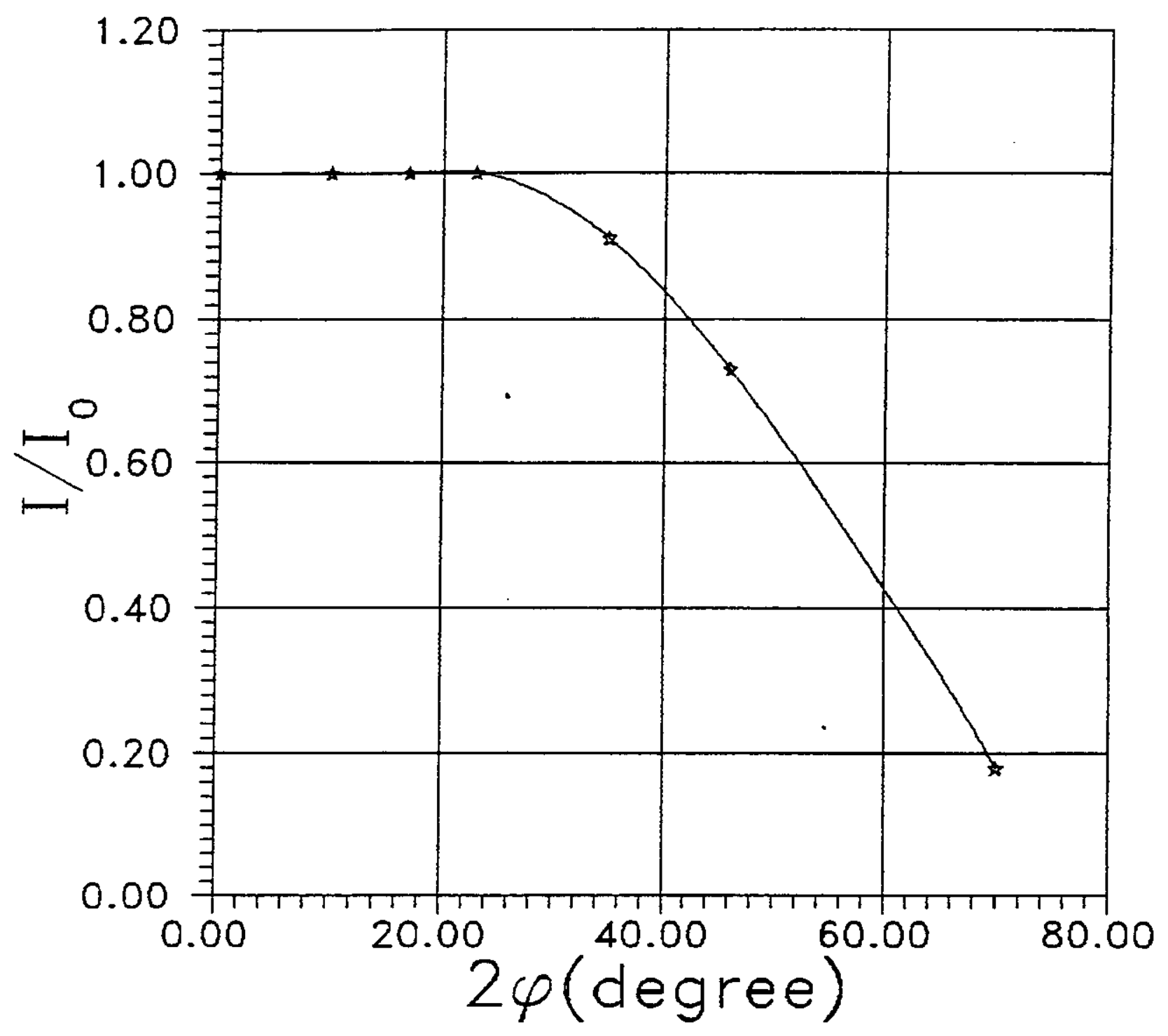

Fig. 2 . 


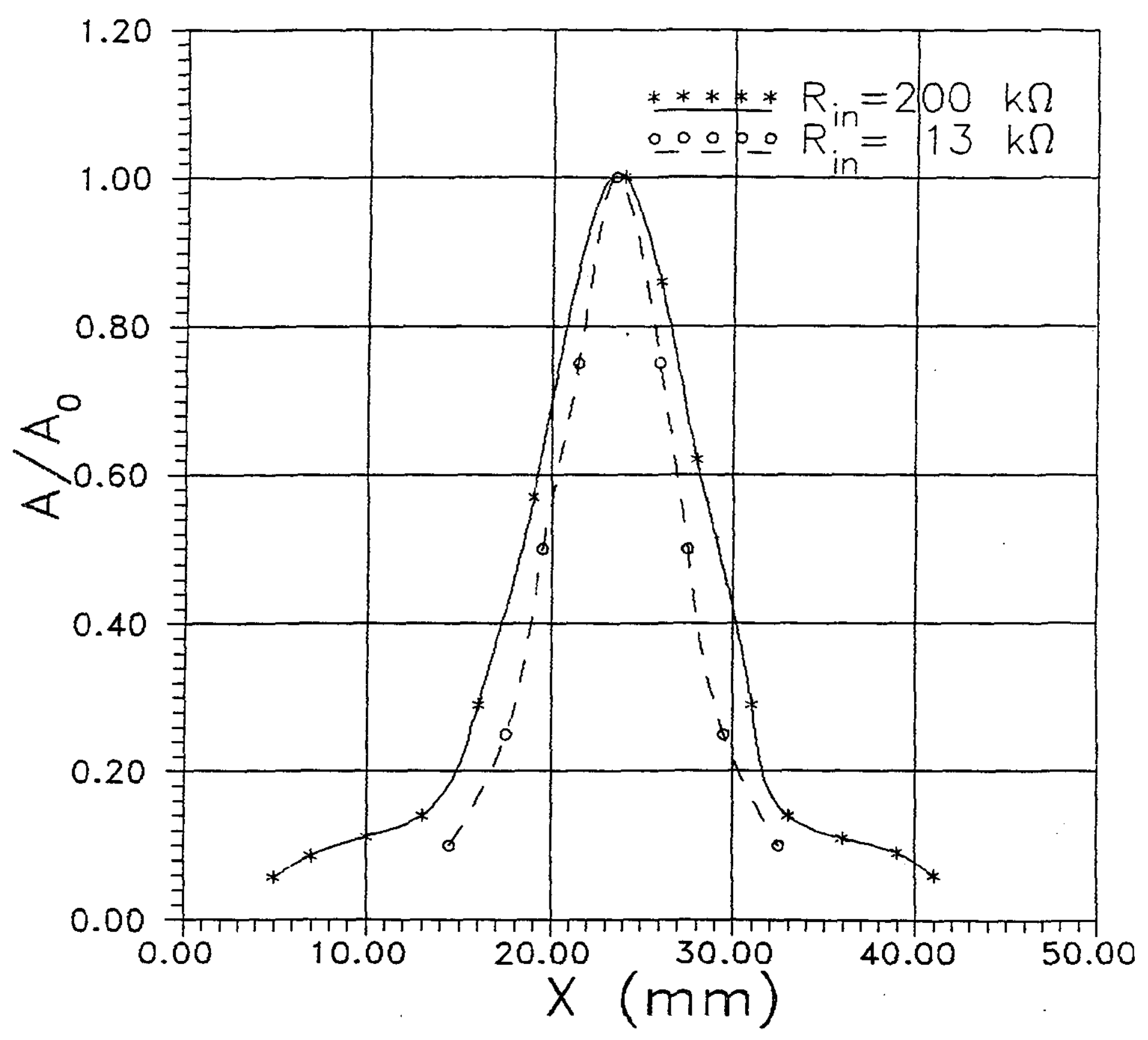

Fig. 3 . 


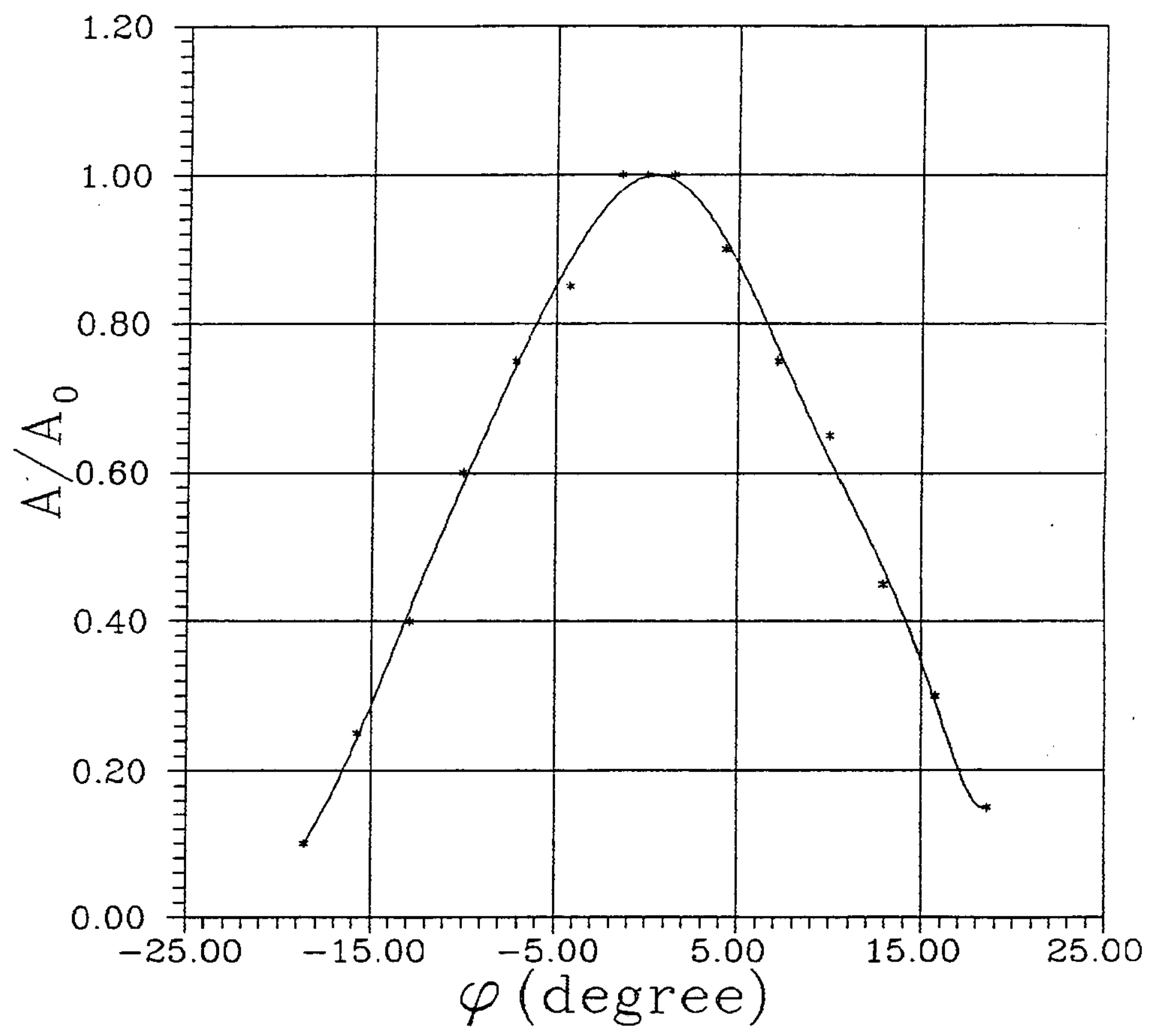

Fig. 4. 

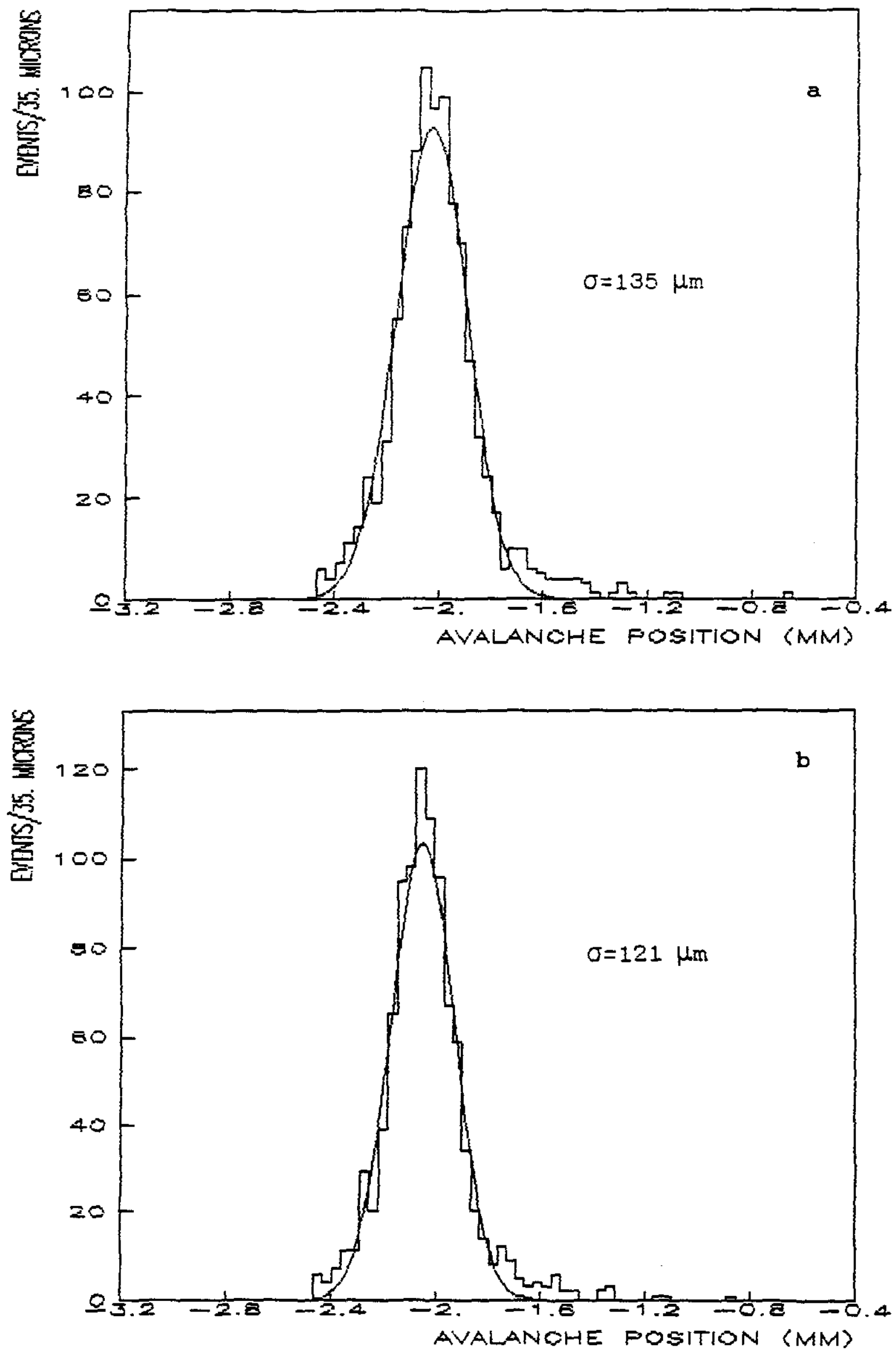

Fi: 5 


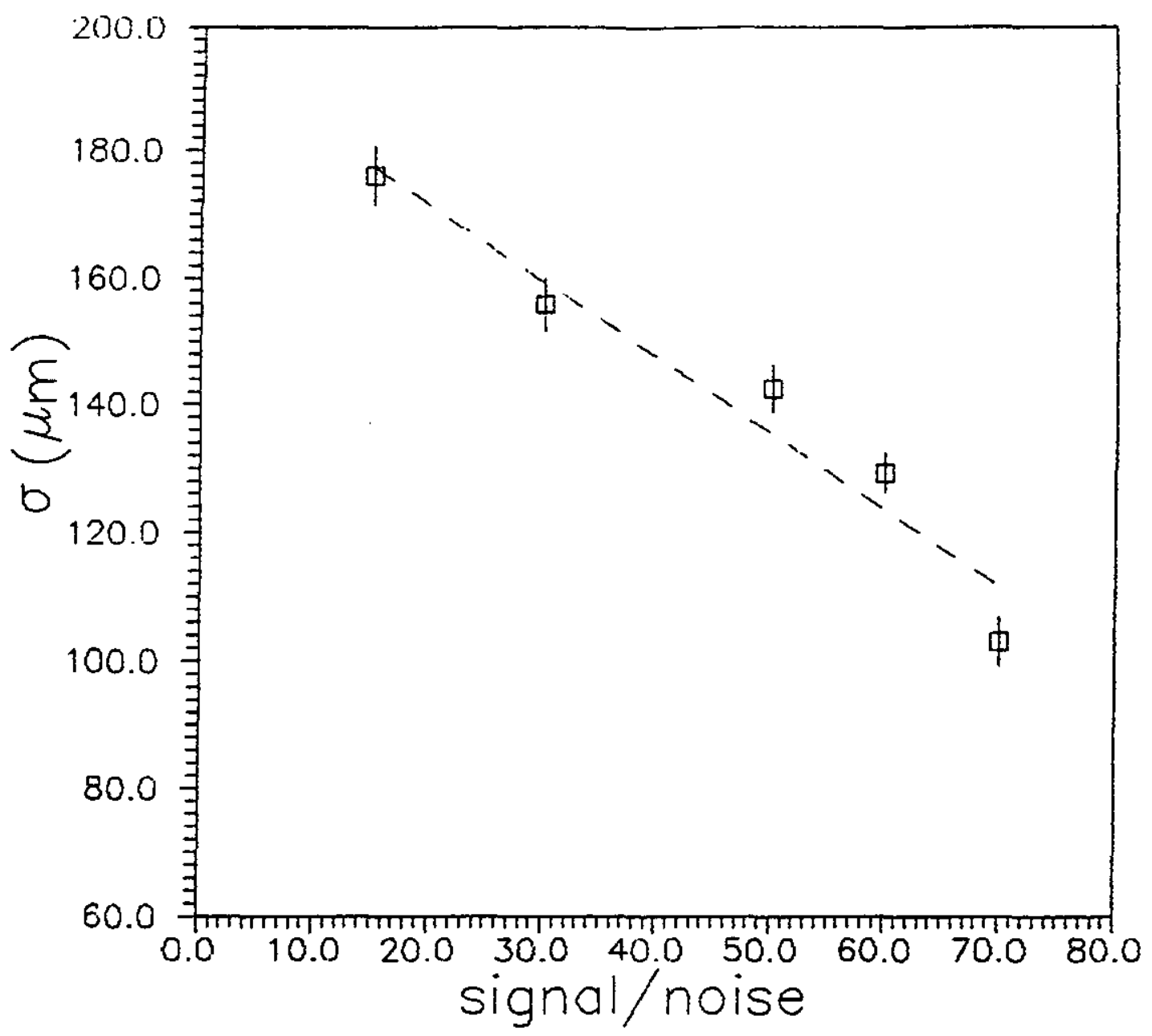

$$
\text { Fig }
$$

